\title{
QUBIT PORTRAIT OF QUDIT STATES AND BELL INEQUALITIES
}

\author{
Vladimir N. Chernega ${ }^{1}$ and Vladimir I. Man'ko ${ }^{2}$ \\ ${ }^{1}$ Faculty of Physics, M.V. Lomonosov Moscow State University \\ Vorob'evy Gory, Moscow 119992, Russia \\ ${ }^{2}$ P.N. Lebedev Physical Institute, Russian Academy of Sciences \\ Leninskii Prospect, 53, Moscow 119991, Russia \\ e-mails: vchernega@gmail.com manko@sci.lebedev.ru
}

\begin{abstract}
A linear map of qudit tomogram onto qubit tomogram (qubit portrait) is proposed as a characteristics of the qudit state. In view of the qubit-portrait method, the Bell inequalities for two qubits and two qutrits are discussed within the framework of the probability-representation of quantum mechanics. A semigroup of stochastic matrices is associated with tomographic-probability distributions of qubit and qutrit states. Bell-like inequalities are studied using the semigroup of stochastic matrices. The qudit-qubit map of tomographic probability distributions is discussed as an ansatz to provide a necessary condition for the separability of quantum states.
\end{abstract}

Keywords: qudits, Bell inequalities, separability, entanglement, qubit portrait, probability representation.

\section{Introduction}

In the probability representation of quantum states [1], the states are described by probability distributions. For example, the spin states are described by probability distribution (called spin tomogram) $w(m, \vec{n})[2,3]$, where $m$ is the spin projection on a direction determined by a unit vector $\vec{n}$. The role of spin tomograms for studing the separability and entanglement of quantum states was pointed out in [4]. Our aim here is to investigate the properties of spin tomograms for one and two spins. Within the quantum-information framework [5], we study qubits and qudits within the context of separable and entangled states. We will obtain that the separable two-qubit states can be associated with $4 \times 4$ stochastic matrices which form a semigroup. This property provides the Bell inequality $[6,7]$ which serves as a criterion of the separability. The Bell inequalities were considered in the context of the probability representation in [8-11]. The probability representation for spin states was discussed and developed in [12-17]. The Shannon entropy [18] of spin state was considered in [19,20]. A linear map of the spintomographic-probability distribution (called qudit tomogram) onto qubit tomogram is constructed. The map provides a qubit portrait of qudit states. The qubit portrait is used to get a necessary condition of the separability of multiqudit states. The preliminary remarks of such map were presented in [21]. Some multiqudit states will be discussed as examples.

The paper is organized as follows.

Manuscript submitted by the authors in English on November 20, 2006.

1573-8760/07/2802-0103 ${ }^{\circledR} 2007$ Springer Science+Business Media, Inc. 
In Sec. 2 we review properties of stochastic matrices. In Sec. 3 we derive an inequality to be used for studying the Bell inequality. In Sec. 4 we consider some matrices as vectors. In Sec. 5 we give a geometrical picture associated with probabilities. In Sec. 6 we present an example of $3 \times 3$ stochastic matrices. In Sec. 7 we show an example of qubit states. In Sec. 8 we discuss entangled two-qubit states. In Sec. 9 we formulate a new separability criterion related to a semigroup of stochastic matrices. In Sec. 10 the new necessary condition of separability is suggested. In Sec. 11 an example of two-qubit entangled state is considered. In Sec. 12 the qubit-portrait method is applied to the qubit-qutrit state. In Sec. 13 a concrete example is given, while Sec. 14, the general reduction criterion of separability is formulated. In Sec. 15 conclusions and perspectives are discussed.

\section{Qubits and Stochastic Matrices}

For one qubit (or for the spin-1/2 particle state) any state vector $|\psi\rangle$ has the form

$$
|\psi\rangle=\left(\begin{array}{c}
a \\
b
\end{array}\right), \quad\langle\psi|=\left(a^{*}, b^{*}\right),
$$

where the complex numbers $a=a_{1}+i a_{2}$ and $b=b_{1}+i b_{2}$ satisfy the normalization condition

$$
\langle\psi \mid \psi\rangle=|a|^{2}+|b|^{2}=1 .
$$

The $2 \times 2$ density matrix of the pure state $|\psi\rangle$ reads

$$
\rho_{\psi}=|\psi\rangle\langle\psi|=\left(\begin{array}{cc}
|a|^{2} & a b^{*} \\
b a^{*} & |b|^{2}
\end{array}\right) .
$$

The trace of the density matrix is

$$
\operatorname{Tr} \rho_{\psi}=|a|^{2}+|b|^{2}=1 .
$$

The diagonal elements of the density matrix determine the probabilities for spin projections on the $z$-axis $m=+1 / 2$ and $m=-1 / 2$, i.e.,

$$
-w(+1 / 2)=|a|^{2}, \quad w(-1 / 2)=|b|^{2} .
$$

Since the probabilities satisfy condition (2), they can be parameterized as follows:

$$
|a|^{2}=\cos ^{2} \Theta, \quad|b|^{2}=\sin ^{2} \Theta .
$$

Let us introduce the matrix

$$
M=\left(\begin{array}{cc}
p & q \\
1-p & 1-q
\end{array}\right),
$$

where the real numbers $p$ and $q$ satisfy the inequalities

$$
1 \geq p \geq 0, \quad 1 \geq q \geq 0 .
$$


The nonnegative numbers $p, 1-p$ and $q, 1-q$ can be considered as probability distributions. A numerical example of such matrix reads

$$
M_{N}=\left(\begin{array}{cc}
1 / 10 & 2 / 5 \\
9 / 10 & 3 / 5
\end{array}\right)
$$

There are two probability distributions. The first one is $(1 / 10,9 / 10)$ and the second one is $(2 / 5,3 / 5)$. The important property of the set of matrices $M$ is that the product of two matrices of the form (7) has the same form, i.e.,

$$
M_{1} M_{2}=\left(\begin{array}{cc}
p_{1} & q_{1} \\
1-p_{1} & 1-q_{1}
\end{array}\right)\left(\begin{array}{cc}
p_{2} & q_{2} \\
1-p_{2} & 1-q_{2}
\end{array}\right)=\left(\begin{array}{cc}
p_{3} & q_{3} \\
1-p_{3} & 1-q_{3}
\end{array}\right),
$$

where

$$
p_{3}=p_{1} p_{2}+q_{1}\left(1-p_{2}\right), \quad q_{3}=p_{1} q_{2}+q_{1}\left(1-q_{2}\right) .
$$

The set of matrices (7) forms a semigroup. The unit matrix belongs to the set. The inverse matrix

$$
M^{-1}=\frac{1}{\operatorname{det} M}\left(\begin{array}{cc}
1-q & -q \\
p-1 & p
\end{array}\right), \quad \operatorname{det} M=p(1-q)-q(1-p),
$$

does not satisfy the condition (8) and does not belong to the set of matrices (7). If parameters $p$ and $q$ are arbitrary real numbers, the set of matrices $M$ with $\operatorname{det} M \neq 0$ provides the two-dimensional Lie group. If parameters $p$ and $q$ are complex numbers, the set of matrices $M$ provides the four-dimensional Lie group. The subset of stochastic matrices of the form

$$
N=\left(\begin{array}{cc}
p & 1-p \\
1-p & p
\end{array}\right)
$$

is also the semigroup. In fact,

$$
N_{1} N_{2}=\left(\begin{array}{cc}
p_{1} & 1-p_{1} \\
1-p_{1} & p_{1}
\end{array}\right)\left(\begin{array}{cc}
p_{2} & 1-p_{2} \\
1-p_{2} & p_{2}
\end{array}\right)=\left(\begin{array}{cc}
p_{3} & 1-p_{3} \\
1-p_{3} & p_{3}
\end{array}\right)
$$

where the nonnegative number

$$
p_{3}=p_{1} p_{2}+\left(1-p_{1}\right)\left(1-p_{2}\right)
$$

determines the matrix elements of matrix

$$
N_{3}=\left(\begin{array}{cc}
p_{3} & 1-p_{3} \\
1-p_{3} & p_{3}
\end{array}\right)
$$

Formula (15) can be used to define the new associative star-product of real (or complex) numbers $p_{1} \star p_{2}$. The set of matrices (13) is called the semigroup of bistochastic matrices. The semigroup can be embedded into the Lie group of complex matrices with complex parameters $p$. The sum of numbers both in columns and rows of bistochastic matrices is equal to one. The bistochastic matrices can be associated with $n \times n$ unitary matrices $u$ with matrix elements $u_{j k}$ satisfying the condition

$$
\sum_{k=1}^{n}\left|u_{j k}\right|^{2}=1, \quad \sum_{j=1}^{n}\left|u_{j k}\right|^{2}=1 .
$$


Thus the stochastic matrix $\wp$ with matrix elements

$$
\wp_{j k}=\left|u_{j k}\right|^{2}
$$

is the bistochastic matrix. This means that the group $u(n)$ of unitary $n \times n$ matrices induces the semigroup of bistochastic matrices (17). The tensor product of two bistochastic matrices is a bistochastic matrix. Thus the group of tensor product of unitary matrices $u\left(n_{1}\right) \otimes u\left(n_{2}\right)$ creates the semigroup, which is the tensor product of bistochastic matrices $\wp_{1} \otimes \wp_{2}$ with matrix elements $\left|u\left(n_{1}\right)_{j k}\right|^{2}$ and $\left|u\left(n_{2}\right)_{\alpha \beta}\right|^{2}$. In view of the property (14), one can introduce the associative product of probability distributions. In fact, given two probability distributions $p_{1}, 1-p_{1}$ and $p_{2}, 1-p_{2}$, one can associate with the probability distributions two vectors

$$
\begin{aligned}
& \overrightarrow{w_{1}}=\left(\begin{array}{c}
p_{1} \\
1-p_{1}
\end{array}\right) \equiv\left(\begin{array}{c}
w_{1}^{(1)} \\
w_{2}^{(1)}
\end{array}\right), \\
& \overrightarrow{w_{2}}=\left(\begin{array}{c}
p_{2} \\
1-p_{2}
\end{array}\right) \equiv\left(\begin{array}{c}
w_{1}^{(2)} \\
w_{2}^{(2)}
\end{array}\right)
\end{aligned}
$$

and two matrices

$$
\begin{aligned}
& N_{1}=\left(\begin{array}{ll}
w_{1}^{(1)} & w_{2}^{(1)} \\
w_{2}^{(1)} & w_{1}^{(1)}
\end{array}\right), \\
& N_{2}=\left(\begin{array}{ll}
w_{1}^{(2)} & w_{2}^{(2)} \\
w_{2}^{(2)} & w_{1}^{(2)}
\end{array}\right) .
\end{aligned}
$$

We define the associative product $\overrightarrow{w_{3}}$ of two vectors (called the star-product) $\overrightarrow{w_{1}} * \overrightarrow{w_{2}}=\overrightarrow{w_{3}}$ using the result of multiplication of two matrices $N_{1}$ and $N_{2}$ given by (14) and (15) to find the components of the vector $\overrightarrow{w_{3}}$. We obtain

$$
\begin{aligned}
& w_{1}^{(3)}=w_{1}^{(1)} w_{1}^{(2)}+w_{2}^{(1)} w_{2}^{(2)}, \\
& w_{2}^{(3)}=w_{2}^{(1)} w_{1}^{(2)}+w_{1}^{(1)} w_{2}^{(2)} .
\end{aligned}
$$

This result can be generalized by introducing the associative product by means of the same tools for $N$-dimensional vectors. The components of the product vector read

$$
p_{m}=\sum_{k=1}^{N} w_{[k+m-1]_{N}} W_{k},
$$

where $[k+m-1]_{N}$ means the number

$$
\begin{cases}k+m-1, & \text { if } \quad k+m-1<N \\ k+m-1-N, & \text { if } \quad k+m-1>N\end{cases}
$$

The eigenvalues of the stochastic matrix (7) are

$$
\lambda_{1}=1, \quad \lambda_{2}=p-q .
$$

They satisfy the condition

$$
\left|\lambda_{k}\right| \leq 1, \quad k=1,2 .
$$


The eigenvectors of the stochastic matrix (7) read

$$
\left|U_{1}\right\rangle=\left(\begin{array}{c}
1 \\
q^{-1}(1-p)
\end{array}\right), \quad\left|U_{p-q}\right\rangle=\left(\begin{array}{c}
-1 \\
1
\end{array}\right)
$$

This means that the matrix $M$ can be presented in the form

$$
\left(\begin{array}{cc}
p & q \\
1-p & 1-q
\end{array}\right)=U\left(\begin{array}{cc}
1 & 0 \\
0 & p-q
\end{array}\right) U^{-1}
$$

where the matrix $U$ reads

$$
U=\left(\begin{array}{cc}
1 & 1 \\
q^{-1}(1-p) & -1
\end{array}\right)
$$

In the case $p=q$, the determinant of the stochastic matrix equals zero. The inverse matrix has the form

$$
U^{-1}=\frac{1}{1+q^{-1}(1-p)}\left(\begin{array}{cc}
1 & 1 \\
q^{-1}(1-p) & -1
\end{array}\right) .
$$

This means that

$$
U^{-1}=U \frac{1}{1+q^{-1}(1-p)}
$$

and

$$
U^{2}=\left(1+q^{-1}(1-p)\right)\left(\begin{array}{ll}
1 & 0 \\
0 & 1
\end{array}\right)
$$

From this property follows

$$
\begin{gathered}
U^{2 k}=\left(1+q^{-1}(1-p)\right)^{k}\left(\begin{array}{cc}
1 & 0 \\
0 & 1
\end{array}\right) \\
U^{2 k+1}=\left(1+q^{-1}(1-p)\right)^{k} U .
\end{gathered}
$$

We obtain from (30)

$$
\left(\begin{array}{cc}
p & q \\
1-p & 1-q
\end{array}\right)^{n}=U\left(\begin{array}{cc}
1 & 0 \\
0 & (p-q)^{n}
\end{array}\right) U^{-1}, \quad n=1,2,3, \ldots
$$

Since $|p-q| \leq 1$, for large $n$ one has $|(p-q)|^{n} \ll 1$. In this case,

$$
\left(\begin{array}{cc}
1 & 0 \\
0 & (p-q)^{n}
\end{array}\right) \longrightarrow\left(\begin{array}{ll}
1 & 0 \\
0 & 0
\end{array}\right)
$$




\section{Useful Inequality}

Now we prove a useful inequality for scalar product of two pairs of real vectors. Let

$$
\left|\left(\overrightarrow{a_{1}} \overrightarrow{b_{1}}\right)\right|<c \text { and }\left|\left(\overrightarrow{a_{2}} \overrightarrow{b_{2}}\right)\right|<c,
$$

where $c$ is a positive number. Then the convex sum $\cos ^{2} \gamma\left(\overrightarrow{a_{1}} \overrightarrow{b_{1}}\right)+\sin ^{2} \gamma\left(\overrightarrow{a_{2}} \overrightarrow{b_{2}}\right)$ satisfies the inequality

$$
\left|\cos ^{2} \gamma\left(\overrightarrow{a_{1}} \overrightarrow{b_{1}}\right)+\sin ^{2} \gamma\left(\overrightarrow{a_{2}} \overrightarrow{b_{2}}\right)\right|<c .
$$

By induction, we get the inequality for generic convex sum. If $\left|\overrightarrow{a_{k}} \overrightarrow{b_{k}}\right|<c$, then

$$
\left|\sum_{k} p_{k}\left(\overrightarrow{a_{k}} \overrightarrow{b_{k}}\right)\right|<c,
$$

where the coefficients

$$
1 \geq p_{k} \geq 0, \quad \sum_{k} p_{k}=1
$$

In particular, we get the following inequality. If $\overrightarrow{b_{1}}=\overrightarrow{b_{2}}=\cdots=\overrightarrow{b_{k}}=\cdots=\vec{B}$, the property (41) reads

$$
\left|\sum_{k} p_{k}\left(\overrightarrow{a_{k}} \vec{B}\right)\right|<c,
$$

i.e.,

$$
\left|\sum_{k}\left(p_{k} \overrightarrow{a_{k}}\right) \vec{B}\right|<c
$$

\section{Matrices as Vectors}

Below we discuss the well-known properties of matrices, namely, how matrices can be interpreted as vectors. For example, the real $2 \times 2$ matrix

$$
\mu=\left(\begin{array}{ll}
a & b \\
c & d
\end{array}\right)
$$

can be considered as the vector

$$
\vec{\mu}=\left(\begin{array}{c}
a \\
b \\
c \\
d
\end{array}\right)
$$


The sum of two matrices $\mu_{1}$ and $\mu_{2}$

$$
\mu_{1}+\mu_{2}=\left(\begin{array}{cc}
a_{1}+a_{2} & b_{1}+b_{2} \\
c_{1}+c_{2} & d_{1}+d_{2}
\end{array}\right)
$$

can be interpreted as the sum of two vectors with the following components:

$$
\overrightarrow{\mu_{1}}+\overrightarrow{\mu_{2}}=\left(\begin{array}{c}
a_{1}+a_{2} \\
b_{1}+b_{2} \\
c_{1}+c_{2} \\
d_{1}+d_{2}
\end{array}\right)
$$

Then the number $\operatorname{Tr}\left(\mu_{1}^{\mathrm{tr}} \mu_{2}\right)=a_{1} a_{2}+b_{1} b_{2}+c_{1} c_{2}+d_{1} d_{2}$, where $\mu_{1}^{\mathrm{tr}}$ is transposed matrix $\mu_{1}$, is the standard scalar product of two vectors, i.e.,

$$
\operatorname{Tr}\left(\mu_{1}^{\mathrm{tr}} \mu_{2}\right)=\left(\overrightarrow{\mu_{1}} \overrightarrow{\mu_{2}}\right)
$$

Let us make a remark. The stochastic matrix $M(7)$ becomes a new stochastic matrix $M^{\prime}$, if one permutes columns of the matrix $M$, i.e.,

$$
M^{\prime}=\left(\begin{array}{cc}
q & p \\
1-q & 1-p
\end{array}\right) \text {. }
$$

The same property takes place if one permutes rows of the matrix $M$. In this case, we get a new stochastic matrix

$$
M^{\prime \prime}=\left(\begin{array}{cc}
1-p & 1-q \\
p & q
\end{array}\right) .
$$

\section{Geometrical Picture}

The probabilities $1 \geq w_{1} \geq 0$ and $1 \geq w_{2} \geq 0$ such that $w_{1}+w_{2}=1$ can be considered in geometrical terms as points on a simplex which is the line shown in Fig. 1.

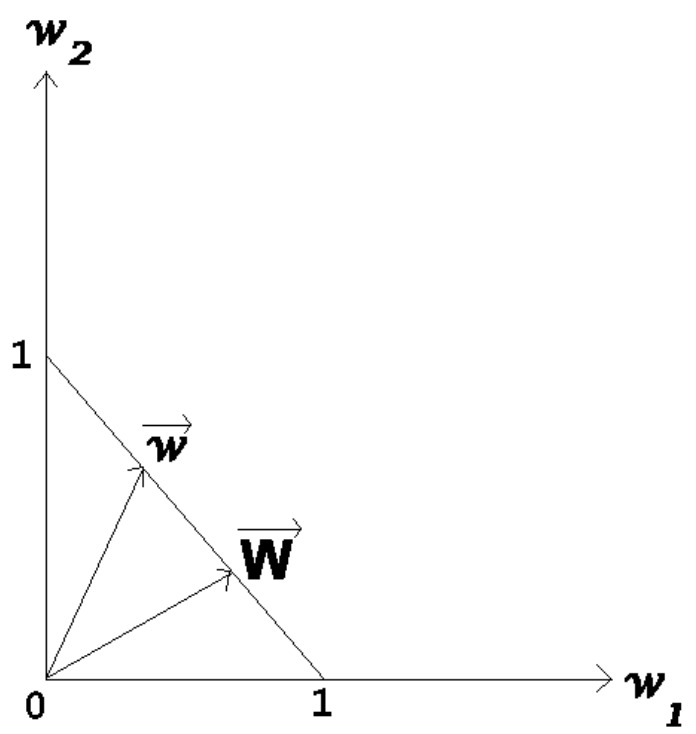

As an example, we show a vector with its end posed on $1 \geq w_{2} \geq 0$. the line that can be given as the column

$$
\vec{w}=\left(\begin{array}{c}
w_{1} \\
w_{2}
\end{array}\right)
$$

The stochastic matrices transform the vector $\vec{w}$ into another vector $\vec{W}$, for example,

$$
\vec{W}=M \vec{w} .
$$


One can check that the vector components

$$
\left(\begin{array}{l}
W_{1} \\
W_{2}
\end{array}\right)=\left(\begin{array}{cc}
q & p \\
1-q & 1-p
\end{array}\right)\left(\begin{array}{l}
w_{1} \\
w_{2}
\end{array}\right)
$$

satisfy the conditions $1 \geq W_{1} \geq 0, \quad 1 \geq W_{2} \geq 0$, and $W_{1}+W_{2}=1$. This means that the stochastic matrices move the initial point on the simplex into another point on the same simplex.

The new probability distribution described by the vector $\vec{W}$ has the components

$$
W_{1}=q w_{1}+p w_{2} \quad \text { and } \quad W_{2}=(1-q) w_{1}+(1-p) w_{2} .
$$

For bistochastic matrices, one has the transformation

$$
W_{1}=q w_{1}+(1-q) w_{2} \quad \text { and } \quad W_{2}=(1-q) w_{1}+q w_{2} .
$$

The point $w_{1}=1 / 2, w_{2}=1 / 2$ is an invariant under this action. For distributions with three components, the simplex has the geometrical form of the plane shown in Fig. 2.

All points on the triangle shown in Fig. 2 correspond to all probability distributions with three outputs.

Below we discuss stochastic matrices which transform a point on this simplex into another point on the same simplex.

\section{6. $3 \times 3$ Stochastic Matrices and Linear Maps of Distributions}

Let us discuss now the third-order stochastic matrices of the form

$$
M=\left(\begin{array}{lll}
p_{1} & q_{1} & r_{1} \\
p_{2} & q_{2} & r_{2} \\
p_{3} & q_{3} & r_{3}
\end{array}\right)
$$

where the positive numbers $p_{k}, q_{k}$, and $r_{k}(k=1,2,3)$ satisfy the normalization condition:

$$
\sum_{k=1}^{3} p_{k}=\sum_{k=1}^{3} q_{k}=\sum_{k=1}^{3} r_{k}=1 .
$$

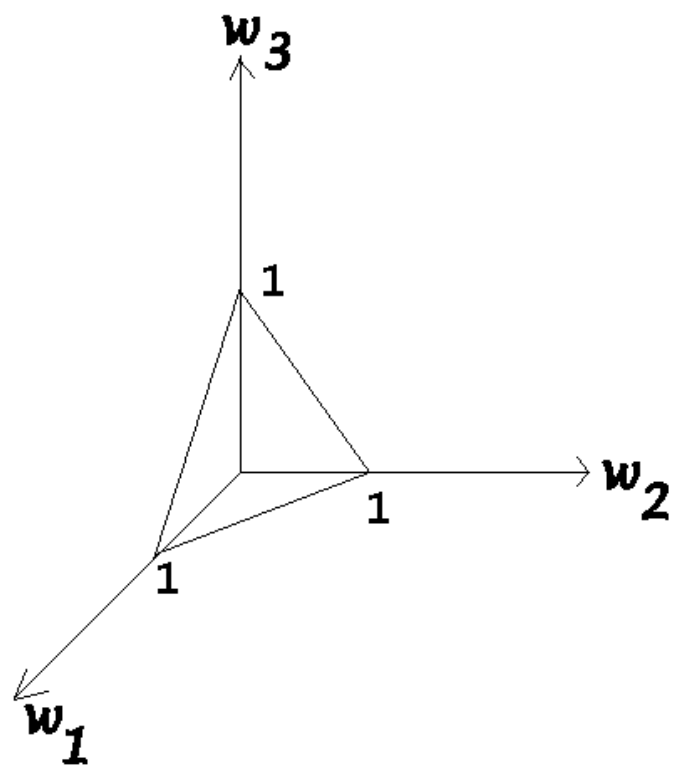

Fig. 2. Simplex of probabilities $w_{1}+w_{2}+w_{3} \leq 1$, all values are nonnegative.

This means that the numbers in columns of the matrix $M$ can be interpreted as probability distributions.

It is easy to check that the set of all matrices $M$ (55) form a semigroup.

Let us give a numerical example of such matrix, i.e.,

$$
M=\left(\begin{array}{ccc}
1 / 10 & 1 / 3 & 8 / 10 \\
3 / 10 & 0 & 1 / 10 \\
6 / 10 & 2 / 3 & 1 / 10
\end{array}\right) .
$$


It is worth noting that the eigenvalues of stochastic matrix $M$ contain $\lambda_{1}=1$. Stochastic matrices $M_{N}$ of all dimensions $N \geq 2$ have eigenvalues equal to unity. One can see that other eigenvalues of stochastic matrix $M_{N}$ can be either real or complex. Also all eigenvalues of stochastic matrices $M_{N}$ satisfy the inequality $\left|\lambda_{k}\right| \leq 1, \quad k=1,2, \ldots, N$.

We point out that the permutations of elements of a chosen column transform a stochastic matrix into another stochastic matrix. The group of all permutations of matrix elements of stochastic matrix $M_{N}$ has $(N !)^{N+1}$ symmetry elements. The group elements are independent permutations in each column $(N !)^{N}$ combined with $N$ ! permutation of columns. The trace of stochastic matrix $M_{N}$ satisfies inequality $\operatorname{Tr} M_{N} \leq N$. The bistochastic $3 \times 3$ matrices have the form (55) but satisfy the extra condition $p_{k}+q_{k}+r_{k}=1(k=1,2,3)$.

The stochastic and bistochastic matrices discussed move the points on the triangle. The point with components $(1 / 3,1 / 3,1 / 3)$ is invariant under the action of bistochastic matrices.

Let us consider the first column of $3 \times 3$ stochastic matrix (55). The nonnegative matrix elements in this column $p_{1}, p_{2}$, and $p_{3}$ can be mapped onto three pairs of nonnegative numbers:

$$
\begin{aligned}
& P_{1}^{(1)}=p_{1}, \quad P_{2}^{(1)}=\left(p_{2}+p_{3}\right), \\
& P_{1}^{(2)}=p_{1}+p_{2}, \quad P_{2}^{(2)}=p_{3}, \\
& P_{1}^{(3)}=p_{1}+p_{3}, \quad P_{2}^{(3)}=p_{2} .
\end{aligned}
$$

Thus we get three probability distributions $\left(P_{1}^{(1)}, P_{2}^{(1)}\right),\left(P_{1}^{(2)}, P_{2}^{(1)}\right)$ and $\left(P_{1}^{(3)}, P_{2}^{(3)}\right)$ and the distributions obtained by permutations of these numbers.

It is easy to see that we constructed the linear map of the initial probability distribution with three possible outcomes onto a set of probability distributions with two outcomes. The map is invertible. In fact,

$$
p_{1}=P_{1}^{(1)}, \quad p_{2}=P_{1}^{(2)}-P_{1}^{(1)}, \quad p_{3}=P_{2}^{(2)} .
$$

This means that if two probability distributions (58)-(60) are known,the initial-probability distribution can be reconstructed. We call the set of probability distributions (58) and (59) "the qubit portrait" of the initial qutrit distribution. We introduce this terminology because we apply the map constructed to study the necessary conditions of separability for quantum multiqudit states. Using the ansatz suggested, one can construct an analogous map for obtaining analogous portraits of joint probability distributions.

\section{Qubit}

If one takes a convex sum of pure state density matrices, the density matrix of mixed state of spin- $1 / 2$ particle (or qubit state) can be obtained. This means that the matrix

$$
\rho=\sum_{k} p_{k}\left|\psi_{k}\right\rangle\left\langle\psi_{k}\right|
$$

where $1 \geq p_{k} \geq 0$ and $\sum_{k} p_{k}=1$ is Hermitian matrix

$$
\rho^{+}=\rho
$$


and its trace is equal to unity. The density matrix is nonnegative matrix, i.e., its eigenvalues are nonnegative numbers. The tomogram of the qubit state is defined by formula

$$
w(m, U)=\left(\begin{array}{c}
w(+1 / 2, U) \\
w(-1 / 2, U)
\end{array}\right)=\left(U^{+} \rho U\right)_{m m},
$$

where $U$ is unitary matrix. It has the form

$$
U=\left(\begin{array}{cc}
\cos \theta / 2 e^{i(\varphi+\psi) / 2} & \sin \theta / 2 e^{i(\varphi-\psi) / 2} \\
-\sin \theta / 2 e^{-i(\varphi-\psi) / 2} & \cos \theta / 2 e^{-i(\varphi+\psi) / 2}
\end{array}\right),
$$

and $\varphi, \theta$, and $\psi$ are Euler angles. In reality, the Euler angle $\psi$ is not present in the final expression of tomogram. The tomogram is the probability distribution. In our previous notation, we can introduce the stochastic matrix using the substitutions

$$
p=w\left(+1 / 2, U_{1}\right), \quad q=w\left(+1 / 2, U_{2}\right),
$$

i.e.,

$$
M=\left(\begin{array}{cc}
w\left(+1 / 2, U_{1}\right) & w\left(+1 / 2, U_{2}\right) \\
1-w\left(+1 / 2, U_{1}\right) & 1-w\left(+1 / 2, U_{2}\right)
\end{array}\right),
$$

where the matrix $U_{1}$ is determined by the angels $\varphi_{1}, \theta_{1}$, and $\psi_{1}$ and the matrix $U_{2}$ is determined by the angels $\varphi_{2}, \theta_{2}$, and $\psi_{2}$. The constructed stochastic matrix with matrix elements equal to tomographic probabilities has all the properties of stochastic matrices (7) discussed in the previous sections.

\section{Two Qubits: Separable and Entangled States}

Let us introduce a unit vector $\vec{n}=(\sin \theta \cos \varphi, \sin \theta \sin \varphi, \cos \theta)$, which is the orthogonal vector to the sphere surface. The tomogram $w(m, U)$ can be considered as a function on the sphere

$$
w(m, U) \equiv w(m, \vec{n})
$$

The stochastic matrix $M$ can be rewritten in the form

$$
M=\left(\begin{array}{cc}
w\left(+1 / 2, \overrightarrow{n_{1}}\right) & w\left(+1 / 2, \overrightarrow{n_{2}}\right) \\
1-w\left(+1 / 2, \overrightarrow{n_{1}}\right) & 1-w\left(+1 / 2, \overrightarrow{n_{2}}\right)
\end{array}\right) .
$$

One has

$$
\begin{aligned}
& w\left(-1 / 2, \overrightarrow{n_{1}}\right)=1-w\left(+1 / 2, \overrightarrow{n_{1}}\right), \\
& w\left(-1 / 2, \overrightarrow{n_{2}}\right)=1-w\left(+1 / 2, \overrightarrow{n_{2}}\right) .
\end{aligned}
$$

Now we consider two qubits. This means that we consider the $4 \times 4$ density matrix $\rho$. The tomogram of two-qubit state reads

$$
w\left(m_{1}, m_{2}, \vec{n}, \vec{N}\right)=\left(U^{\dagger} \rho U\right)_{m_{1} m_{2}, m_{1} m_{2}},
$$


where $U$ is the $4 \times 4$ unitary matrix, which is the tensor product of two $2 \times 2$ unitary matrices

$$
U=U_{1} \otimes U_{2}
$$

with $U_{1}$ and $U_{2}$ given by formula (65) with the Euler angels $\varphi_{1} \theta_{1} \psi_{1}$ and $\varphi_{2} \theta_{2} \psi_{2}$, respectively. The vector $\vec{n}$ is determined by the Euler angels $\varphi_{1}, \theta_{1}$ and the vector $\vec{N}$ is determined by the Euler angels $\varphi_{2}, \theta_{2}$.

A simply separable state has the tomogram of a factorized form

$$
w\left(m_{1}, m_{2}, \vec{n}, \vec{N}\right)=w_{1}\left(m_{1}, \vec{n}\right) w_{2}\left(m_{2}, \vec{N}\right)
$$

Now we construct the $4 \times 4$ stochastic matrix following the rule.

We take four vectors $\vec{a}, \vec{b}, \vec{c}$, and $\vec{d}$. Then we choose two vectors $\vec{n}$ to be equal to $\vec{a}$ and $\vec{b}$ and two vectors $\vec{N}$ to be equal to $\vec{c}$ and $\vec{d}$. We have two probability distributions for the first qubit $w_{1}\left(m_{1}, \vec{a}\right)$ and $w_{1}\left(m_{1}, \vec{b}\right)$, and two probability distributions for the second qubit $w_{2}\left(m_{2}, \vec{c}\right)$ and $w_{2}\left(m_{2}, \vec{d}\right)$. Then our $4 \times 4$ stochastic matrix reads

$$
\begin{aligned}
\left(M_{4}\right)_{k 1}= & \left(\begin{array}{l}
w_{1}(+1 / 2, \vec{a}) w_{2}(+1 / 2, \vec{b}) \\
w_{1}(+1 / 2, \vec{a}) w_{2}(-1 / 2, \vec{b}) \\
w_{1}(-1 / 2, \vec{a}) w_{2}(+1 / 2, \vec{b}) \\
w_{1}(-1 / 2, \vec{a}) w_{2}(-1 / 2, \vec{b})
\end{array}\right), \quad k=1,2,3,4, \\
\left(M_{4}\right)_{k 2}= & \left(\begin{array}{l}
w_{1}(+1 / 2, \vec{a}) w_{2}(+1 / 2, \vec{c}) \\
w_{1}(+1 / 2, \vec{a}) w_{2}(-1 / 2, \vec{c}) \\
w_{1}(-1 / 2, \vec{a}) w_{2}(+1 / 2, \vec{c}) \\
w_{1}(-1 / 2, \vec{a}) w_{2}(-1 / 2, \vec{c})
\end{array}\right), \quad k=1,2,3,4, \\
\left(M_{4}\right)_{k 3}= & \left(\begin{array}{l}
w_{1}(+1 / 2, \vec{d}) w_{2}(+1 / 2, \vec{b}) \\
w_{1}(+1 / 2, \vec{d}) w_{2}(-1 / 2, \vec{b}) \\
w_{1}(-1 / 2, \vec{d}) w_{2}(+1 / 2, \vec{b}) \\
w_{1}(-1 / 2, \vec{d}) w_{2}(-1 / 2, \vec{b})
\end{array}\right), \quad k=1,2,3,4,
\end{aligned}
$$

and

$$
\left(M_{4}\right)_{k 4}=\left(\begin{array}{l}
w_{1}(+1 / 2, \vec{d}) w_{2}(+1 / 2, \vec{c}) \\
w_{1}(+1 / 2, \vec{d}) w_{2}(-1 / 2, \vec{c}) \\
w_{1}(-1 / 2, \vec{d}) w_{2}(+1 / 2, \vec{c}) \\
w_{1}(-1 / 2, \vec{d}) w_{2}(-1 / 2, \vec{c})
\end{array}\right), \quad k=1,2,3,4
$$

This matrix can be presented in the form of tensor product of two stochastic $2 \times 2$ matrices, i.e.,

$$
M_{4}=\left(\begin{array}{ll}
w_{1}(+1 / 2, \vec{a}) & w_{1}(+1 / 2, \vec{d}) \\
w_{1}(-1 / 2, \vec{a}) & w_{1}(-1 / 2, \vec{d})
\end{array}\right) \otimes\left(\begin{array}{ll}
w_{2}(+1 / 2, \vec{b}) & w_{2}(+1 / 2, \vec{c}) \\
w_{2}(-1 / 2, \vec{b}) & w_{2}(-1 / 2, \vec{c})
\end{array}\right) .
$$


We call this stochastic matrix "simply separable stochastic matrix." One can check that matrix (77) satisfies the Bell-Clauser-Horne-Shimony-Holt inequality (so-called Bell-CHSH inequality) [7]

$$
\begin{aligned}
& \mid\left(M_{4}\right)_{11}-\left(M_{4}\right)_{21}-\left(M_{4}\right)_{31}+\left(M_{4}\right)_{41}+\left(M_{4}\right)_{12}-\left(M_{4}\right)_{22}-\left(M_{4}\right)_{32}+\left(M_{4}\right)_{42} \\
& +\left(M_{4}\right)_{13}-\left(M_{4}\right)_{23}-\left(M_{4}\right)_{33}+\left(M_{4}\right)_{43}-\left(M_{4}\right)_{14}+\left(M_{4}\right)_{24}+\left(M_{4}\right)_{34}-\left(M_{4}\right)_{44} \mid \leq 2 .
\end{aligned}
$$

This inequality can be rewritten in the matrix form as $\left|\operatorname{Tr}\left(M_{4} I\right)\right| \leq 2$, where

$$
I=\left(\begin{array}{cccc}
1 & -1 & -1 & 1 \\
1 & -1 & -1 & 1 \\
1 & -1 & -1 & 1 \\
-1 & 1 & 1 & -1
\end{array}\right)
$$

The inequality has to be preserved, if one changes the matrix $I$ by the product matrix $\tilde{I}=I C$, where $C=C_{1} \otimes C_{2}$. Here two $2 \times 2$ matrices $C_{1}$ and $C_{2}$ are arbitrary stochastic matrices. In vector form, $M_{4} \rightarrow \overrightarrow{M_{4}}$ and, according to the rules of Sec. 3, $I \rightarrow \vec{I}$, this inequality reads

$$
\left|\left(\vec{I} \overrightarrow{M_{4}}\right)\right| \leq 2 \text {. }
$$

Due to the property of convex sums (41), one can state that if one constructs a convex sum of matrices of the type $M_{4}$

$$
M=\sum_{k} P_{k} M_{4}^{(k)}, \quad P_{k} \geq 0, \quad \sum_{k} P_{k}=1,
$$

the inequality

$$
|\vec{I} \vec{M}| \leq 2
$$

or

$$
|\operatorname{Tr}(M I)| \leq 2
$$

can be obtained.

\section{Separable and Entangled States}

By definition, the quantum state of two qubits is separable, if the state tomogram can be presented in the form of convex sum of simply separable tomograms, i.e.,

$$
w\left(m_{1} m_{1} \overrightarrow{n_{1}} \overrightarrow{n_{2}}\right)=\sum_{k} P_{k} w_{1}^{(k)}\left(m_{1} \overrightarrow{n_{1}}\right) w_{2}^{(k)}\left(m_{2} \overrightarrow{n_{2}}\right), \quad P_{k} \geq 0, \quad \sum_{k} P_{k}=1,
$$

where the index $k$ can be understood as a collective index with any number of components including both discrete and continuous ones. One can see that the stochastic matrix corresponding to tomogram (84) has the form of the convex sum of matrices of the type (77), i.e.,

$$
M_{4}=\sum_{k} P_{k}\left(\begin{array}{ll}
w^{(k)}(+1 / 2, \vec{a}) & w^{(k)}(+1 / 2, \vec{d}) \\
w^{(k)}(-1 / 2, \vec{a}) & w^{(k)}(-1 / 2, \vec{d})
\end{array}\right) \otimes\left(\begin{array}{ll}
w^{(k)}(+1 / 2, \vec{b}) & w^{(k)}(+1 / 2, \vec{c}) \\
w^{(k)}(-1 / 2, \vec{b}) & w^{(k)}(-1 / 2, \vec{c})
\end{array}\right) .
$$


We call this stochastic matrix the separable stochastic matrix.

\section{Lemma}

The product of two stochastic matrices $M_{4}^{(1)}$ and $M_{4}^{(2)}$ corresponding to tomograms of separable states of two qubits is the convex sum of simply separable stochastic matrices.

\section{Proof}

Let $\overline{F_{1} \text { be }}$ stochastic matrix corresponding to a separable two-qubit quantum state, i.e., it can be written in the form (85) which we denote as

$$
F_{1}=\sum_{k} P_{k} w_{(1)}^{(k)}
$$

where

$$
w_{(1)}^{(k)}=\left(\begin{array}{ll}
w^{(k)}\left(+1 / 2, \overrightarrow{a_{1}}\right) & w^{(k)}\left(+1 / 2, \overrightarrow{d_{1}}\right) \\
w^{(k)}\left(-1 / 2, \overrightarrow{a_{1}}\right) & w^{(k)}\left(-1 / 2, \overrightarrow{d_{1}}\right)
\end{array}\right) \otimes\left(\begin{array}{cc}
w^{(k)}\left(+1 / 2, \overrightarrow{b_{1}}\right) & w^{(k)}\left(+1 / 2, \overrightarrow{c_{1}}\right) \\
w^{(k)}\left(-1 / 2, \overrightarrow{b_{1}}\right) & w^{(k)}\left(-1 / 2, \overrightarrow{c_{1}}\right)
\end{array}\right) .
$$

Let $F_{2}$ be another stochastic matrix of the form

$$
F_{2}=\sum_{s} \rho_{s} w_{(2)}^{(s)}
$$

where $\rho_{s} \geq 0$ and $\sum_{s} \rho_{s}=1$. The notation (88) means that we change in (87)

$$
k \rightarrow s, \quad \overrightarrow{a_{1}} \rightarrow \overrightarrow{a_{2}}, \quad \overrightarrow{d_{1}} \rightarrow \overrightarrow{d_{2}}, \quad \overrightarrow{b_{1}} \rightarrow \overrightarrow{b_{2}}, \quad \overrightarrow{c_{1}} \rightarrow \overrightarrow{c_{2}}
$$

Now we calculate the product matrix

$$
F=F_{1} F_{2}=\sum_{k s}\left(P_{k} \rho_{s}\right) w_{(1)}^{(k)} w_{(2)}^{(s)}
$$

Since the rule of multiplication of tensor products of matrices reads

$$
(a \otimes b)(c \otimes d)=(a c) \otimes(b d),
$$

one has

$$
F=\sum_{j} Q_{j} w^{j}
$$

where $j$ is a collective index $j=(k s)$, the matrix $w^{(j)}$ is the $4 \times 4$ stochastic matrix of simply separable form. This means that the matrix $F$ satisfies the Bell-CHSH inequality

$$
|\operatorname{Tr}(F I)| \leq 2
$$




\section{Necessary Condition of Separability}

The lemma proved above is used to formulate the necessary condition of separability of a two-qubit state. In fact, if one has the two-qubit separable state with spin tomogram $w\left(m_{1} m_{2} \overrightarrow{n_{1}} \overrightarrow{n_{2}}\right)$, the set of matrices associated with the tomogram, in view of the rule

$$
M(\vec{a} \vec{b} \vec{c} \vec{d})=\left(\begin{array}{cccc}
w\left(+\frac{1}{2} \vec{a}+\frac{1}{2} \vec{b}\right) & w\left(+\frac{1}{2} \vec{a}+\frac{1}{2} \vec{c}\right) & w\left(+\frac{1}{2} \vec{d}+\frac{1}{2} \vec{b}\right) & w\left(+\frac{1}{2} \vec{d}+\frac{1}{2} \vec{c}\right) \\
w\left(+\frac{1}{2} \vec{a}-\frac{1}{2} \vec{b}\right) & w\left(+\frac{1}{2} \vec{a}-\frac{1}{2} \vec{c}\right) & w\left(+\frac{1}{2} \vec{d}-\frac{1}{2} \vec{b}\right) & w\left(+\frac{1}{2} \vec{d}-\frac{1}{2} \vec{c}\right) \\
w\left(-\frac{1}{2} \vec{a}+\frac{1}{2} \vec{b}\right) & w\left(-\frac{1}{2} \vec{a}+\frac{1}{2} \vec{c}\right) & w\left(-\frac{1}{2} \vec{d}+\frac{1}{2} \vec{b}\right) & w\left(-\frac{1}{2} \vec{d}+\frac{1}{2} \vec{c}\right) \\
w\left(-\frac{1}{2} \vec{a}-\frac{1}{2} \vec{b}\right) & w\left(-\frac{1}{2} \vec{a}-\frac{1}{2} \vec{c}\right) & w\left(-\frac{1}{2} \vec{d}-\frac{1}{2} \vec{b}\right) & w\left(-\frac{1}{2} \vec{d}-\frac{1}{2} \vec{c}\right)
\end{array}\right),
$$

forms the semigroup of matrices satisfying inequality (83). This property can be used as a criterion of the separability.

For example, we take the two matrices $M_{1}\left(\overrightarrow{a_{1}} \overrightarrow{b_{1}} \overrightarrow{c_{1}} \overrightarrow{d_{1}}\right)$ and $M_{2}\left(\overrightarrow{a_{2}} \overrightarrow{b_{2}} \overrightarrow{c_{2}} \overrightarrow{d_{2}}\right)$. We check that for both matrices the product

$$
F=M_{1} M_{2}\left(\overrightarrow{a_{1}} \overrightarrow{b_{1}} \overrightarrow{c_{1}} \overrightarrow{d_{1}} \overrightarrow{a_{2}} \overrightarrow{b_{2}} \overrightarrow{c_{2}} \overrightarrow{d_{2}}\right)
$$

satisfies inequality (83) for arbitrary directions $\left(\overrightarrow{a_{k}} \overrightarrow{b_{k}} \overrightarrow{c_{k}} \overrightarrow{d_{k}}\right)$ with $k=1,2$.

This property can be generalized to any number of directions $k=1,2, \ldots$

It is worth noting that the product of two density matrices of two separable quantum states is not the density matrix of a quantum state.

\section{Example of Entangled States}

Let us consider a known example of entangled state of two qubits

$$
\rho=\frac{1}{2}\left(\begin{array}{llll}
1 & 0 & 0 & 1 \\
0 & 0 & 0 & 0 \\
0 & 0 & 0 & 0 \\
1 & 0 & 0 & 1
\end{array}\right)
$$

We construct the tomogram of this state using the matrices (71) and (72). The result reads

$$
\begin{aligned}
w\left(+\frac{1}{2},+\frac{1}{2}, \overrightarrow{n_{1}}, \overrightarrow{n_{2}}\right) & =\frac{1}{2}\left(\cos ^{2} \frac{\Theta_{1}}{2} \cos ^{2} \frac{\Theta_{2}}{2}+\sin ^{2} \frac{\Theta_{1}}{2} \sin ^{2} \frac{\Theta_{2}}{2}\right)+\frac{1}{4} \sin \Theta_{1} \sin \Theta_{2} \cos \left(\varphi_{1}+\varphi_{2}\right), \\
w\left(+\frac{1}{2},-\frac{1}{2}, \overrightarrow{n_{1}}, \overrightarrow{n_{2}}\right) & =\frac{1}{2}\left(\cos ^{2} \frac{\Theta_{1}}{2} \sin ^{2} \frac{\Theta_{2}}{2}+\sin ^{2} \frac{\Theta_{1}}{2} \cos ^{2} \frac{\Theta_{2}}{2}\right)-\frac{1}{4} \sin \Theta_{1} \sin \Theta_{2} \cos \left(\varphi_{1}+\varphi_{2}\right), \\
w\left(-\frac{1}{2},+\frac{1}{2}, \overrightarrow{n_{1}}, \overrightarrow{n_{2}}\right) & =\frac{1}{2}\left(\cos ^{2} \frac{\Theta_{1}}{2} \sin ^{2} \frac{\Theta_{2}}{2}+\sin ^{2} \frac{\Theta_{1}}{2} \cos ^{2} \frac{\Theta_{2}}{2}\right)-\frac{1}{4} \sin \Theta_{1} \sin \Theta_{2} \cos \left(\varphi_{1}+\varphi_{2}\right), \\
w\left(-\frac{1}{2},-\frac{1}{2}, \overrightarrow{n_{1}}, \overrightarrow{n_{2}}\right) & =\frac{1}{2}\left(\cos ^{2} \frac{\Theta_{1}}{2} \cos ^{2} \frac{\Theta_{2}}{2}+\sin ^{2} \frac{\Theta_{1}}{2} \sin ^{2} \frac{\Theta_{2}}{2}\right)+\frac{1}{4} \sin \Theta_{1} \sin \Theta_{2} \cos \left(\varphi_{1}+\varphi_{2}\right) .
\end{aligned}
$$


The matrix $M(\vec{a}, \vec{b}, \vec{c}, \vec{d})$ associated with tomogram (95) has 16 matrix elements:

$$
\begin{aligned}
& M_{11}=\frac{1}{2}\left(\cos ^{2} \frac{\Theta_{a}}{2} \cos ^{2} \frac{\Theta_{b}}{2}+\sin ^{2} \frac{\Theta_{a}}{2} \sin ^{2} \frac{\Theta_{b}}{2}\right)+\frac{1}{4} \sin \Theta_{a} \sin \Theta_{b} \cos \left(\varphi_{a}+\varphi_{b}\right) \text {, } \\
& M_{21}=\frac{1}{2}\left(\cos ^{2} \frac{\Theta_{a}}{2} \sin ^{2} \frac{\Theta_{b}}{2}+\sin ^{2} \frac{\Theta_{a}}{2} \cos ^{2} \frac{\Theta_{b}}{2}\right)-\frac{1}{4} \sin \Theta_{a} \sin \Theta_{b} \cos \left(\varphi_{a}+\varphi_{b}\right) \text {, } \\
& M_{31}=\frac{1}{2}\left(\cos ^{2} \frac{\Theta_{a}}{2} \sin ^{2} \frac{\Theta_{b}}{2}+\sin ^{2} \frac{\Theta_{a}}{2} \cos ^{2} \frac{\Theta_{b}}{2}\right)-\frac{1}{4} \sin \Theta_{a} \sin \Theta_{b} \cos \left(\varphi_{a}+\varphi_{b}\right) \text {, } \\
& M_{41}=\frac{1}{2}\left(\cos ^{2} \frac{\Theta_{a}}{2} \cos ^{2} \frac{\Theta_{b}}{2}+\sin ^{2} \frac{\Theta_{a}}{2} \sin ^{2} \frac{\Theta_{b}}{2}\right)+\frac{1}{4} \sin \Theta_{a} \sin \Theta_{b} \cos \left(\varphi_{a}+\varphi_{b}\right) \text {, } \\
& M_{12}=\frac{1}{2}\left(\cos ^{2} \frac{\Theta_{a}}{2} \cos ^{2} \frac{\Theta_{c}}{2}+\sin ^{2} \frac{\Theta_{a}}{2} \sin ^{2} \frac{\Theta_{c}}{2}\right)+\frac{1}{4} \sin \Theta_{a} \sin \Theta_{c} \cos \left(\varphi_{a}+\varphi_{c}\right) \text {, } \\
& M_{22}=\frac{1}{2}\left(\cos ^{2} \frac{\Theta_{a}}{2} \sin ^{2} \frac{\Theta_{c}}{2}+\sin ^{2} \frac{\Theta_{a}}{2} \cos ^{2} \frac{\Theta_{c}}{2}\right)-\frac{1}{4} \sin \Theta_{a} \sin \Theta_{c} \cos \left(\varphi_{a}+\varphi_{c}\right) \text {, } \\
& M_{32}=\frac{1}{2}\left(\cos ^{2} \frac{\Theta_{a}}{2} \sin ^{2} \frac{\Theta_{c}}{2}+\sin ^{2} \frac{\Theta_{a}}{2} \cos ^{2} \frac{\Theta_{c}}{2}\right)-\frac{1}{4} \sin \Theta_{a} \sin \Theta_{c} \cos \left(\varphi_{a}+\varphi_{c}\right) \text {, } \\
& M_{42}=\frac{1}{2}\left(\cos ^{2} \frac{\Theta_{a}}{2} \cos ^{2} \frac{\Theta_{c}}{2}+\sin ^{2} \frac{\Theta_{a}}{2} \sin ^{2} \frac{\Theta_{c}}{2}\right)+\frac{1}{4} \sin \Theta_{a} \sin \Theta_{c} \cos \left(\varphi_{a}+\varphi_{c}\right) \text {, } \\
& M_{13}=\frac{1}{2}\left(\cos ^{2} \frac{\Theta_{d}}{2} \cos ^{2} \frac{\Theta_{b}}{2}+\sin ^{2} \frac{\Theta_{d}}{2} \sin ^{2} \frac{\Theta_{b}}{2}\right)+\frac{1}{4} \sin \Theta_{d} \sin \Theta_{b} \cos \left(\varphi_{d}+\varphi_{b}\right) \text {, } \\
& M_{23}=\frac{1}{2}\left(\cos ^{2} \frac{\Theta_{d}}{2} \sin ^{2} \frac{\Theta_{b}}{2}+\sin ^{2} \frac{\Theta_{d}}{2} \cos ^{2} \frac{\Theta_{b}}{2}\right)-\frac{1}{4} \sin \Theta_{d} \sin \Theta_{b} \cos \left(\varphi_{d}+\varphi_{b}\right) \text {, } \\
& M_{33}=\frac{1}{2}\left(\cos ^{2} \frac{\Theta_{d}}{2} \sin ^{2} \frac{\Theta_{b}}{2}+\sin ^{2} \frac{\Theta_{d}}{2} \cos ^{2} \frac{\Theta_{b}}{2}\right)-\frac{1}{4} \sin \Theta_{d} \sin \Theta_{b} \cos \left(\varphi_{d}+\varphi_{b}\right) \text {, } \\
& M_{43}=\frac{1}{2}\left(\cos ^{2} \frac{\Theta_{d}}{2} \cos ^{2} \frac{\Theta_{b}}{2}+\sin ^{2} \frac{\Theta_{d}}{2} \sin ^{2} \frac{\Theta_{b}}{2}\right)+\frac{1}{4} \sin \Theta_{d} \sin \Theta_{b} \cos \left(\varphi_{d}+\varphi_{b}\right) \text {, } \\
& M_{14}=\frac{1}{2}\left(\cos ^{2} \frac{\Theta_{d}}{2} \cos ^{2} \frac{\Theta_{c}}{2}+\sin ^{2} \frac{\Theta_{d}}{2} \sin ^{2} \frac{\Theta_{c}}{2}\right)+\frac{1}{4} \sin \Theta_{d} \sin \Theta_{c} \cos \left(\varphi_{d}+\varphi_{c}\right), \\
& M_{24}=\frac{1}{2}\left(\cos ^{2} \frac{\Theta_{d}}{2} \sin ^{2} \frac{\Theta_{c}}{2}+\sin ^{2} \frac{\Theta_{d}}{2} \cos ^{2} \frac{\Theta_{c}}{2}\right)-\frac{1}{4} \sin \Theta_{d} \sin \Theta_{c} \cos \left(\varphi_{d}+\varphi_{c}\right) \text {, } \\
& M_{34}=\frac{1}{2}\left(\cos ^{2} \frac{\Theta_{d}}{2} \sin ^{2} \frac{\Theta_{c}}{2}+\sin ^{2} \frac{\Theta_{d}}{2} \cos ^{2} \frac{\Theta_{c}}{2}\right)-\frac{1}{4} \sin \Theta_{d} \sin \Theta_{c} \cos \left(\varphi_{d}+\varphi_{c}\right) \text {, } \\
& M_{44}=\frac{1}{2}\left(\cos ^{2} \frac{\Theta_{d}}{2} \cos ^{2} \frac{\Theta_{c}}{2}+\sin ^{2} \frac{\Theta_{d}}{2} \sin ^{2} \frac{\Theta_{c}}{2}\right)+\frac{1}{4} \sin \Theta_{d} \sin \Theta_{c} \cos \left(\varphi_{d}+\varphi_{c}\right) \text {. }
\end{aligned}
$$

One can see that the matrix $M(96)$ violates the condition (83), which is the Bell inequality, for some angles and takes the maximum value $2 \sqrt{2}$, which is the Cirelson bound [22]. This is due to the entanglement of state (94). Violation of the Bell inequalities is a signal that the state is entangled. The product $M$ of two matrices (96) corresponding to angles $\Theta_{a}, \Theta_{b}, \Theta_{c}, \Theta_{d}, \varphi_{a}, \varphi_{b}, \varphi_{c}$, and $\varphi_{d}$ for the first matrix $M_{1}$ and $\Theta_{a^{\prime}}, \Theta_{b^{\prime}}, \Theta_{c^{\prime}}, \Theta_{d^{\prime}}, \varphi_{a^{\prime}}, \varphi_{b^{\prime}}, \varphi_{c^{\prime}}$, and $\varphi_{d^{\prime}}$ for the second matrix $M_{2}$, i.e., $M=M_{1} M_{2}$ must satisfy the Bell inequality (83) for a separable state. These matrices form a semigroup which is the sub-semigroup 
of all stochastic matrices constructed by means of tomograms of all quantum states.

\section{Reduction of the Qubit-Qutrit Separability Property to Bell Inequalities for Two Qubits.}

In this section, we demonstrate the new necessary condition of separability of qubit-qutrit state using the probability representation of quantum states. The idea of the construction is to find the qubit portrait of the qutrit state discussed in the previous sections. If one has the probability-distribution vector with three nonnegative components

$$
\vec{W}=\left(\begin{array}{l}
W_{1} \\
W_{2} \\
W_{3}
\end{array}\right)
$$

where $W_{1}+W_{2}+W_{3}=1$, a new probability-distribution vector $\vec{\rho}$ can be constructed as follows:

$$
\vec{\rho}=\left(\begin{array}{c}
\rho_{1} \\
\rho_{2}
\end{array}\right)=\left(\begin{array}{c}
W_{1} \\
W_{2}+W_{3}
\end{array}\right) .
$$

This means that each three-dimensional distribution induces two-dimensional ones. One can use all vectors

$$
\overrightarrow{\rho^{\prime}}=\left(\begin{array}{c}
\rho_{1}^{\prime} \\
\rho_{2}^{\prime}
\end{array}\right)=\left(\begin{array}{c}
W_{1}+W_{2} \\
W_{3}
\end{array}\right)
$$

and

$$
\overrightarrow{\rho^{\prime \prime}}=\left(\begin{array}{c}
\rho_{1}^{\prime \prime} \\
\rho_{2}^{\prime \prime}
\end{array}\right)=\left(\begin{array}{c}
W_{1}+W_{3} \\
W_{2}
\end{array}\right)
$$

Let us consider a simply separable state of the qubit-qutrit system with the density operator

$$
\hat{\rho}(1,2)=\hat{\rho}(1) \otimes \hat{\rho}(2) .
$$

Then the tomogram of this state is the probability distribution of the form

$$
w\left(m_{1}, \overrightarrow{n_{1}}, m_{2}, \overrightarrow{n_{2}}\right)=w_{1}\left(m_{1}, \overrightarrow{n_{1}}\right) W\left(m_{2}, \overrightarrow{n_{2}}\right),
$$

where the spin projection $m_{1}$ take values $-1 / 2$ and $+1 / 2$ and the spin projection $m_{2}$ takes values $-1,1$, and 0 .

In the form of a six-dimensional vector, tomogram (101) can be rewritten as follows:

$$
\vec{w}\left(\overrightarrow{n_{1}}, \overrightarrow{n_{2}}\right)=\overrightarrow{w_{1 / 2}}\left(\overrightarrow{n_{1}}\right) \otimes \overrightarrow{W_{1}}\left(\overrightarrow{n_{2}}\right)
$$

where

$$
\overrightarrow{w_{1 / 2}}=\left(\begin{array}{l}
w_{1}\left(\overrightarrow{n_{1}}\right) \\
w_{2}\left(\overrightarrow{n_{1}}\right)
\end{array}\right)
$$


and

$$
\overrightarrow{W_{1}}\left(\overrightarrow{n_{2}}\right)=\left(\begin{array}{l}
W_{1}\left(\overrightarrow{n_{2}}\right) \\
W_{2}\left(\overrightarrow{n_{2}}\right) \\
W_{3}\left(\overrightarrow{n_{2}}\right)
\end{array}\right)
$$

Thus one has

$$
\vec{w}\left(\overrightarrow{n_{1}}, \overrightarrow{n_{2}}\right)=\left(\begin{array}{l}
w_{1}\left(\overrightarrow{n_{1}}\right) W_{1}\left(\overrightarrow{n_{2}}\right) \\
w_{1}\left(\overrightarrow{n_{1}}\right) W_{2}\left(\overrightarrow{n_{2}}\right) \\
w_{1}\left(\overrightarrow{n_{1}}\right) W_{3}\left(\overrightarrow{n_{2}}\right) \\
w_{2}\left(\overrightarrow{n_{1}}\right) W_{1}\left(\overrightarrow{n_{2}}\right) \\
w_{2}\left(\overrightarrow{n_{1}}\right) W_{2}\left(\overrightarrow{n_{2}}\right) \\
w_{2}\left(\overrightarrow{n_{1}}\right) W_{3}\left(\overrightarrow{n_{2}}\right)
\end{array}\right)
$$

Now we apply the described ansatz of reduction of the three-dimensional distributions to the twodimensional ones. From (104), we obtain the vector

$$
\overrightarrow{\rho_{1}}\left(\overrightarrow{n_{2}}\right)=\left(\begin{array}{c}
W_{1}\left(\overrightarrow{n_{2}}\right) \\
W_{2}\left(\overrightarrow{n_{2}}\right)+W_{3}\left(\overrightarrow{n_{2}}\right)
\end{array}\right)
$$

This reduction induces the reduction of the six-vector (105) to the four-vector

$$
\vec{\rho}\left(\overrightarrow{n_{1}}, \overrightarrow{n_{2}}\right)=\left(\begin{array}{c}
w_{1}\left(\overrightarrow{n_{1}}\right) W_{1}\left(\overrightarrow{n_{2}}\right) \\
w_{1}\left(\overrightarrow{n_{1}}\right)\left(W_{2}\left(\overrightarrow{n_{2}}\right)+W_{3}\left(\overrightarrow{n_{2}}\right)\right) \\
w_{2}\left(\overrightarrow{n_{1}}\right) W_{1}\left(\overrightarrow{n_{2}}\right) \\
w_{2}\left(\overrightarrow{n_{1}}\right)\left(W_{2}\left(\overrightarrow{n_{2}}\right)+W_{3}\left(\overrightarrow{n_{2}}\right)\right)
\end{array}\right) .
$$

One has the simple observation. If a tomogram is simply separable, the reduced distribution vector $\vec{\rho}\left(\overrightarrow{n_{1}}, \overrightarrow{n_{2}}\right)$ is also simply separable distribution. From this property follows the same property for a convex sum of simply separable distributions.

One has for a separable quantum state of the qubit-qutrit system the following property of its spin tomogram.

Let the spin tomogram be given by a probability distribution $w\left(m_{1}, \overrightarrow{n_{1}}, m_{2}, \overrightarrow{n_{2}}\right)$, which corresponds either to separable or entangled state.

Let us denote this tomogram by the vector

$$
\vec{w}\left(\overrightarrow{n_{1}}, \overrightarrow{n_{2}}\right)=\left(\begin{array}{c}
w\left(+1 / 2, \overrightarrow{n_{1}},+1, \overrightarrow{n_{2}}\right) \\
w\left(+1 / 2, \overrightarrow{n_{1}}, 0, \overrightarrow{n_{2}}\right) \\
w\left(+1 / 2, \overrightarrow{n_{1}},-1, \overrightarrow{n_{2}}\right) \\
w\left(-1 / 2, \overrightarrow{n_{1}},+1, \overrightarrow{n_{2}}\right) \\
w\left(-1 / 2, \overrightarrow{n_{1}}, 0, \overrightarrow{n_{2}}\right) \\
w\left(-1 / 2, \overrightarrow{n_{1}},-1, \overrightarrow{n_{2}}\right)
\end{array}\right) .
$$


Then we introduce the four-vector

$$
\vec{\rho}\left(\overrightarrow{n_{1}}, \overrightarrow{n_{2}}\right)=\left(\begin{array}{c}
w\left(+1 / 2, \overrightarrow{n_{1}},+1, \overrightarrow{n_{2}}\right) \\
w\left(+1 / 2, \overrightarrow{n_{1}}, 0, \overrightarrow{n_{2}}\right)+w\left(+1 / 2, \overrightarrow{n_{1}},-1, \overrightarrow{n_{2}}\right) \\
w\left(-1 / 2, \overrightarrow{n_{1}},+1, \overrightarrow{n_{2}}\right) \\
w\left(-1 / 2, \overrightarrow{n_{1}}, 0, \overrightarrow{n_{2}}\right)+w\left(-1 / 2, \overrightarrow{n_{1}},-1, \overrightarrow{n_{2}}\right)
\end{array}\right) .
$$

Now we apply the criterion of separability discussed in the previous sections and used for two-qubit states. This means that we construct stochastic $4 \times 4$ matrix where in the column there are the components of vectors (109) with the corresponding vectors $\overrightarrow{n_{1}}$ and $\overrightarrow{n_{2}}$

$$
P(\vec{a}, \vec{b}, \vec{c}, \vec{d})=\|\vec{\rho}(\vec{a}, \vec{b}) \vec{\rho}(\vec{a}, \vec{c}) \vec{\rho}(\vec{d}, \vec{b}) \vec{\rho}(\vec{d}, \vec{c})\|
$$

We obtain the result.

If the matrix elements of matrix (110) violate the Bell inequality, the qubit-qutrit state is entangled. Fulfilling the Bell inequality (92) is the necessary condition of separability of the qubit-qutrit state.

\section{Qubit-Qutrit and Two Qutrits}

In this section, we present here two examples of entangled states.

Let the density matrix of a qubit-qutrit state in the standard basis $\left|1 / 2, m_{1}\right\rangle\left|1, m_{2}\right\rangle$ have the form

$$
\rho=\frac{1}{2}\left(\begin{array}{llllll}
1 & 0 & 0 & 0 & 0 & 1 \\
0 & 0 & 0 & 0 & 0 & 0 \\
0 & 0 & 0 & 0 & 0 & 0 \\
0 & 0 & 0 & 0 & 0 & 0 \\
0 & 0 & 0 & 0 & 0 & 0 \\
1 & 0 & 0 & 0 & 0 & 1
\end{array}\right) .
$$

Two unitary matrices transforming qubits

$$
U_{11}=e^{i \varphi_{1} / 2} \cos \frac{\theta_{1}}{2}, \quad U_{12}=i e^{i \varphi_{1} / 2} \sin \frac{\theta_{1}}{2}, \quad U_{21}=i e^{-i \varphi_{1} / 2} \sin \frac{\theta_{1}}{2}, \quad U_{22}=e^{-i \varphi_{1} / 2} \cos \frac{\theta_{1}}{2}
$$

and qutrits

$$
\begin{aligned}
& V_{11}=e^{i \varphi_{2}} \cos ^{2} \frac{\theta_{2}}{2}, \quad V_{12}=i e^{i \varphi_{2}} \frac{\sin \Theta_{2}}{\sqrt{2}}, \quad V_{13}=-e^{i \varphi_{2}} \sin ^{2} \frac{\theta_{2}}{2}, \\
& V_{21}=i \frac{\sin \Theta_{2}}{\sqrt{2}}, \quad V_{22}=\cos \Theta_{2}, \quad V_{23}=i \frac{\sin \Theta_{2}}{\sqrt{2}}, \\
& V_{31}=-e^{-i \varphi_{2}} \sin ^{2} \frac{\theta_{2}}{2}, \quad V_{32}=i e^{-i \varphi_{2}} \frac{\sin \Theta_{2}}{\sqrt{2}}, \quad V_{33}=e^{-i \varphi_{2}} \cos ^{2} \frac{\theta_{2}}{2}
\end{aligned}
$$


can be used to construct the $6 \times 6$ matrices $U \otimes V$ and $U^{\dagger} \otimes V^{\dagger}$. The diagonal matrix elements of the matrix

$$
\left[\left(U^{\dagger} \otimes V^{\dagger}\right) \rho(U \otimes V)\right]_{m_{1} m_{2}, m_{1} m_{2}}=w\left(m_{1}, \overrightarrow{n_{1}}, m_{2}, \overrightarrow{n_{2}}\right)
$$

provide the spin tomogram of state (111). Here the two vectors are determined by angles $\Theta_{1}, \varphi_{1}, \Theta_{2}$, and $\varphi_{2}$ as follows:

$$
\overrightarrow{n_{1}}=\left(\sin \Theta_{1} \cos \varphi_{1}, \sin \Theta_{1} \sin \varphi_{1}, \cos \Theta_{1}\right) \quad \text { and } \quad \overrightarrow{n_{2}}=\left(\sin \Theta_{2} \cos \varphi_{2}, \sin \Theta_{2} \sin \varphi_{2}, \cos \Theta_{2}\right)
$$

One has

$$
\begin{aligned}
w\left(+\frac{1}{2}, \overrightarrow{n_{1}},+1, \overrightarrow{n_{2}}\right) & =\frac{1}{2}\left|U_{11} V_{11}+U_{21} V_{31}\right|^{2}, & w\left(+\frac{1}{2}, \overrightarrow{n_{1}}, 0, \overrightarrow{n_{2}}\right) & =\frac{1}{2}\left|U_{11} V_{12}+U_{21} V_{32}\right|^{2}, \\
w\left(+\frac{1}{2}, \overrightarrow{n_{1}},-1, \overrightarrow{n_{2}}\right) & =\frac{1}{2}\left|U_{11} V_{13}+U_{21} V_{33}\right|^{2}, & w\left(-\frac{1}{2}, \overrightarrow{n_{1}},+1, \overrightarrow{n_{2}}\right) & =\frac{1}{2}\left|U_{12} V_{11}+U_{22} V_{31}\right|^{2}, \\
w\left(-\frac{1}{2}, \overrightarrow{n_{1}}, 0, \overrightarrow{n_{2}}\right) & =\frac{1}{2}\left|U_{12} V_{12}+U_{22} V_{32}\right|^{2}, & w\left(-\frac{1}{2}, \overrightarrow{n_{1}},-1, \overrightarrow{n_{2}}\right) & =\frac{1}{2}\left|U_{12} V_{13}+U_{22} V_{33}\right|^{2} .
\end{aligned}
$$

Applying the reduction ansatz we obtain the $4 \times 4$ matrix (110). By calculating the modulus of trace of product of this matrix and the matrix $I$ given by (79), we get the expression, which we denote as follows:

$$
B=\left|\sin \Theta_{a}\left(\sin ^{2} \Theta_{b} \sin \Phi_{a b}+\sin ^{2} \Theta_{c} \sin \Phi_{a c}\right)+\sin \Theta_{d}\left(\sin ^{2} \Theta_{b} \sin \Phi_{d b}-\sin ^{2} \Theta_{c} \sin \Phi_{d c}\right)\right|,
$$

where

$$
\Phi_{a b}=\varphi_{a}+2 \varphi_{b}, \quad \Phi_{a c}=\varphi_{a}+2 \varphi_{c}, \quad \Phi_{d b}=\varphi_{d}+2 \varphi_{b}, \quad \Phi_{d c}=\varphi_{d}+2 \varphi_{c} .
$$

One can check that for the parameters

$$
\Theta_{a}=\frac{\pi}{2}, \quad \Theta_{b}=\frac{\pi}{2}, \quad \Theta_{c}=\frac{\pi}{2}, \quad \Theta_{d}=\frac{\pi}{2}, \quad \Phi_{a b}=\frac{\pi}{2}, \quad \Phi_{d c}=-\frac{\pi}{4}, \quad \Phi_{a c}=\frac{\pi}{4}, \quad \Phi_{d b}=0,
$$

the value $B(116)$ is larger than 2 , namely,

$$
B=1+\sqrt{2} .
$$

This means that the qubit-qutrit state is entangled. This is clear because the density matrix (111) corresponds to the pure entangled state

$$
|\Psi\rangle=\frac{1}{\sqrt{2}}(|+1 / 2\rangle|+1\rangle+|-1 / 2\rangle|-1\rangle)
$$

For the two-qutrit entangled state with $9 \times 9$ density matrix comtaining 72 matrix elements equal to zero except 9 matrix elements

$$
\rho_{11}=\rho_{15}=\rho_{19}=\rho_{51}=\rho_{55}=\rho_{59}=\rho_{91}=\rho_{95}=\rho_{99}=1 / 3,
$$


the spin tomogram can be calculated by the same method using two $3 \times 3$ matrices $U$ and $V$ given by the same relations (113). But the matrix elements of the matrix $U$ are taken to be dependent on angles $\varphi_{1}$ and $\Theta_{1}$. We get the vector $\vec{w}\left(\overrightarrow{n_{1}}, \overrightarrow{n_{2}}\right)$ with 9 components:

$$
\begin{array}{ll}
w\left(+1, \overrightarrow{n_{1}},+1, \overrightarrow{n_{2}}\right)=\frac{1}{3}\left|\sum_{j=1}^{3} U_{j 1} V_{j 1}\right|^{2}, & w\left(+1, \overrightarrow{n_{1}}, 0, \overrightarrow{n_{2}}\right)=\frac{1}{3}\left|\sum_{j=1}^{3} U_{j 1} V_{j 2}\right|^{2} ; \\
w\left(+1, \overrightarrow{n_{1}},-1, \overrightarrow{n_{2}}\right)=\frac{1}{3}\left|\sum_{j=1}^{3} U_{j 1} V_{j 3}\right|^{2}, & w\left(0, \overrightarrow{n_{1}},+1, \overrightarrow{n_{2}}\right)=\frac{1}{3}\left|\sum_{j=1}^{3} U_{j 2} V_{j 1}\right|^{2}, \\
w\left(0, \overrightarrow{n_{1}}, 0, \overrightarrow{n_{2}}\right)=\frac{1}{3}\left|\sum_{j=1}^{3} U_{j 2} V_{j 2}\right|^{2}, & \\
w\left(0, \overrightarrow{n_{1}},-1, \overrightarrow{n_{2}}\right)=\frac{1}{3}\left|\sum_{j=1}^{3} U_{j 2} V_{j 3}\right|^{2}, & w\left(-1, \overrightarrow{n_{1}},+1, \overrightarrow{n_{2}}\right)=\frac{1}{3}\left|\sum_{j=1}^{3} U_{j 3} V_{j 1}\right|^{2}, \\
w\left(-1, \overrightarrow{n_{1}}, 0, \overrightarrow{n_{2}}\right)=\frac{1}{3}\left|\sum_{j=1}^{3} U_{j 3} V_{j 2}\right|^{2}, & w\left(-1, \overrightarrow{n_{1}},-1, \overrightarrow{n_{2}}\right)=\frac{1}{3}\left|\sum_{j=1}^{3} U_{j 3} V_{j 3}\right|^{2} .
\end{array}
$$

We construct the qubit portrait of this state. One of the four-vectors $\vec{P}\left(\overrightarrow{n_{1}}, \overrightarrow{n_{2}}\right)$ of this portrait has the components

$$
\begin{aligned}
& P_{1}\left(\overrightarrow{n_{1}}, \overrightarrow{n_{2}}\right)=w\left(+1, \overrightarrow{n_{1}},+1, \overrightarrow{n_{2}}\right) \\
& P_{2}\left(\overrightarrow{n_{1}}, \overrightarrow{n_{2}}\right)=w\left(+1, \overrightarrow{n_{1}}, 0, \overrightarrow{n_{2}}\right)+w\left(+1, \overrightarrow{n_{1}},-1, \overrightarrow{n_{2}}\right) \\
& P_{3}\left(\overrightarrow{n_{1}}, \overrightarrow{n_{2}}\right)=w\left(0, \overrightarrow{n_{1}},+1, \overrightarrow{n_{2}}\right)+w\left(-1, \overrightarrow{n_{1}},+1, \overrightarrow{n_{2}}\right) \\
& P_{4}\left(\overrightarrow{n_{1}}, \overrightarrow{n_{2}}\right)=w\left(0, \overrightarrow{n_{1}}, 0, \overrightarrow{n_{2}}\right)+w\left(0, \overrightarrow{n_{1}},-1, \overrightarrow{n_{2}}\right)+w\left(-1, \overrightarrow{n_{1}}, 0, \overrightarrow{n_{2}}\right)+w\left(-1, \overrightarrow{n_{1}},-1, \overrightarrow{n_{2}}\right) .
\end{aligned}
$$

Using (120) and (121) and taking pairs

$$
\overrightarrow{n_{1}}=\vec{a}, \quad \overrightarrow{n_{2}}=\vec{b}, \quad \overrightarrow{n_{1}}=\vec{a}, \quad \overrightarrow{n_{2}}=\vec{c}
$$

and

$$
\overrightarrow{n_{1}}=\vec{d}, \quad \overrightarrow{n_{2}}=\vec{b}, \quad \overrightarrow{n_{1}}=\vec{d}, \quad \overrightarrow{n_{2}}=\vec{c},
$$

one can construct the $4 \times 4$ matrix (110). Calculating the modulus of the trace of the product of matrix (79) with the obtained matrix, we get the value of $B$ of the form

$$
\begin{aligned}
& B=\frac{1}{2} \mid\left[\left(\cos \Theta_{b}+1\right)^{2}-2\right]\left(\cos \Theta_{a}+\cos \Theta_{d}\right)+\left[\left(\cos \Theta_{c}+1\right)^{2}-2\right]\left(\cos \Theta_{a}-\cos \Theta_{d}\right) \\
& -\sin ^{2} \Theta_{b}\left(\sin \Phi_{a b} \sin \Theta_{a}+\sin \Phi_{d b} \sin \Theta_{d}\right)-\sin ^{2} \Theta_{c}\left(\sin \Phi_{a c} \sin \Theta_{a}+\sin \Phi_{d c} \sin \Theta_{d}\right) \mid .
\end{aligned}
$$

One can check that for angles

$$
\varphi_{a}=2 \pi, \quad \varphi_{b}=-\frac{\pi}{8}, \quad \varphi_{c}=\frac{\pi}{8}, \quad \varphi_{d}=0, \quad \Theta_{a}=0, \quad \Theta_{b}=\frac{\pi}{2}, \quad \Theta_{c}=\frac{\pi}{2}, \quad \Theta_{d}=\frac{\pi}{2},
$$

the value of $B$ is $(1+\sqrt{2})>2$. It corresponds to entangled two-qutrit state. 


\section{General Reduction Criterion of Separability}

Now we use the experience with the discussed qubit-qutrit system to formulate a general criterion of separability for a state of bipartite quantum system. The criterion is based on the property of the separable state tomogram of the bipartite system.

For simplicity, we take a two qudit separable state with the tomogram of the form (84). Let us associate with this tomogram the joint probability distribution given as four nonnegative numbers

$$
\begin{aligned}
& \tilde{w}\left(M_{1}=j_{1}, M_{2}=j_{2}, \overrightarrow{n_{1}}, \overrightarrow{n_{2}}\right)=w\left(j_{1}, j_{2}, \overrightarrow{n_{1}}, \overrightarrow{n_{2}}\right), \\
& \tilde{w}\left(M_{1}=j_{1}, M_{2}=j_{2}-1, \overrightarrow{n_{1}}, \overrightarrow{n_{2}}\right)=\sum_{m_{2}=-j_{2}}^{j_{2}-1} w\left(j_{1}, m_{2}, \overrightarrow{n_{1}}, \overrightarrow{n_{2}}\right), \\
& \tilde{w}\left(M_{1}=j_{1}-1, M_{2}=j_{2}, \overrightarrow{n_{1}}, \overrightarrow{n_{2}}\right)=\sum_{m_{1}=-j_{1}}^{j_{1}-1} w\left(m_{1}, j_{2}, \overrightarrow{n_{1}}, \overrightarrow{n_{2}}\right), \\
& \tilde{w}\left(M_{1}=j_{1}-1, M_{2}=j_{2}-1, \overrightarrow{n_{1}}, \overrightarrow{n_{2}}\right)=\sum_{m_{1}=-j_{1}}^{j_{1}-1} \sum_{m_{2}=-j_{2}}^{j_{2}-1} w\left(m_{1}, m_{2}, \overrightarrow{n_{1}}, \overrightarrow{n_{2}}\right),
\end{aligned}
$$

where $M_{1}$ takes the values $j_{1}$ and $j_{1}-1$ and $M_{2}$ takes the values $j_{2}$ and $j_{2}-1$. We reinterpret the obtained joint probability distribution as "a two-qubit tomogram." Due to this, the Bell inequality is fulfilled for the probability distribution, if the initial two-qudit state is separable. We used the ansatz of obtaining the reduced joint probability distribution by summing the probabilities in the initial probability distribution with a larger number of possible events (or measurements). But the separability of the initial quantum state is preserved in the process of such summation in the sense that, if the initial tomographic-probability distribution looks like the convex sum of the product of two distributions, the reduced distribution is also the convex sum of the product of two probability distributions. The result obtained can be formulated as the following reduction criterion of separability.

The necessary condition of separability of a bipartite-system state is the separability property of the reduced state tomogram. Fulfilling the Bell inequalities for reduced state tomogram is a necessary condition of separability of the quantum state under study. One can give a recipe for studying the separability of a given state of a bipartite system. The first step is to obtain the state tomogram. Then one has to reduce this tomogram by summing over all such events to get the "tomogram" of two qubits. Then one checks the fulfillment of the Bell inequality for the reduced tomogram obtained. If it is violated, the initial state is entangled.

\section{Conclusions}

To conclude, we summarize the main results of our work.

We have shown that the qudit states can be mapped onto probability distributions which are the points on the simplex. The probability distributions can be considered as vectors. The stochastic and bistochastic matrices can be constructed using these vectors as columns of the matrices. Both stochastic and bistochastic matrices form semigroups. The invertible map of probability distributions onto bistochastic matrix was used to construct the star-product of the probability distributions. For qudit 
tomograms, we have introduced the notion of qubit portrait. We have shown that the necessary condition of separability of a bipartite qudit state is the separability of its qubit portrait. The violation of the Bell inequality for the qubit portrait of the bipartite-system state (both for qudit states and for continuous variables) means that the system state is entangled. Examples of entangled qubit-qutrit state and two-qutrit state were considered using the method of constructing the qubit portrait of the states. The method can be generalized for multiqudit systems.

\section{References}

1. S. Mancini, V. I. Man'ko, and P. Tombesi, Phys. Lett. A, 213, 1 (1996).

2. V. V. Dodonov and V. I. Man'ko, Phys. Lett. A, 239, 335 (1997).

3. V. I. Man'ko and O. V. Man'ko, JETP, 85, 430 (1997).

4. V. I. Man'ko, G. Marmo, E. C. G. Sudarshan, and F. Zaccaria, Phys. Lett. A, 327, 353 (2004).

5. M. A. Man'ko, V. I. Man'ko, and R. V. Mendes, "A probability operator symbol framework for quantum information"[0602quant-ph/0602189v1], J. Russ. Laser Res., 27, 507 (2006).

6. J. S. Bell, Physics (Long Island City, N. Y.), 1, 195 (1964).

7. J. F. Clauser, M. A. Horne, A. Shimony, and R. A. Holt, Phys. Rev. Lett., 23, 880 (1969).

8. C. Lupo, V. I. Man'ko, and G. Marmo, J. Phys. A: Math. Gen., 39, 12515 (2006).

9. V. A. Andreev and V. I. Man'ko, JETP, 87, 239 (1998).

10. V.A. Andreev and V. I. Man'ko, Teor. Mat. Fiz. , 140, 284 (2004).

11. V. A. Andreev, V. I. Man'ko, O. V. Man'ko, and E. V. Shchukin, Teor. Mat. Fiz., 146, 140 (2006).

12. M. O. Tera-Cunha, V. I. Man'ko, and M. O. Scully, Found. Phys. Lett., 14, 103 (2001).

13. V. I. Man'ko and S. S. Safonov, Phys. At. Nucl., 62, 658 (1998).

14. V. A. Andreev, O. V. Man'ko, V. I. Man'ko, and S. S. Safonov, J. Russ. Laser Res., 19, 340 (1998).

15. O. Castaños, R. Lopés-Peña, M. A. Man'ko, J. Phys. A: Math. Gen., 36, 4677 (2003).

16. A. B. Klimov, O. V. Man'ko, V. I. Man'ko, Yu. F. Smirnov and V. N. Tolstoy, J. Phys. A: Math. Gen., 35, 6101 (2002).

17. O. V. Man'ko, V. I. Man'ko, and G. Marmo, Phys. Scr., 62, 446 (2000).

18. C. E. Shannon, Bell System. Tech. J., 27, 379 (1948).

19. O. V. Man'ko and V. I. Man'ko, "Probability representation entropy for spin-state tomogram" [quant-ph/041131], J. Russ. Laser Res., 25, 115 (2004).

20. V. N. Chernega, O. V. Man'ko, V. I. Man'ko, O. V. Pilyavets, and V. G. Zborovskii, J. Russ. Laser Res., 27, 132 (2006).

21. O. V. Man'ko, V. I. Man'ko, J. Russ. Laser Res., 27, 319 (2006).

22. B. S. Cirel'son, Lett. Math. Phys., 4, 93 (1980). 